\title{
Combining Frontal Gamma Asymmetry Neurofeedback with Virtual Reality: A Proof-of-Concept Case Study
}

\author{
Jeff Tarrant ${ }^{*}$ and Hannah Cope
}

NeuroMeditation Institute, Corvallis, Oregon, USA

\begin{abstract}
The current proof-of-concept case study was designed to determine if a consumer-grade neurofeedback system (Muse) could be used in conjunction with specially designed virtual reality (VR) environments (Positivity by Healium) to impact gamma frontal asymmetry as well as create positive changes in mood states. Four firefighters served as subjects and completed pre-post mood rating scales as well as 19-channel EEG recordings. An examination of sLORETA frontal lobe ROls demonstrated a postintervention gamma asymmetry shift to the left in three of the four subjects. In addition, subjects generally reported changes in mood consistent with the frontal asymmetry changes. Overall, these results provide initial support for the idea that a consumer-grade brain computer interface $(\mathrm{BCl}) / \mathrm{VR}$ intervention can potentially have therapeutic utility and deserve further study.
\end{abstract}

Keywords: virtual reality; positivity; gamma; frontal asymmetry; sLORETA; Muse

Citation: Tarrant, J., \& Cope, H. (2018). Combining frontal gamma asymmetry neurofeedback with virtual reality: A proof-of-concept case study. NeuroRegulation, 5(2), 57-67. http://dx.doi.org/10.15540/nr.5.2.57

*Address correspondence to: Dr. Jeff Tarrant, 873 NW Grant Edited by:

Avenue, Suite A, Corvallis, OR 97330, USA. Email: Rex L. Cannon, PhD, Knoxville Neurofeedback Group, Knoxville, dr.tarrant@hotmail.com

Tennessee, USA

Copyright: (c) 2018. Tarrant and Cope. This is an Open Access article distributed under the terms of the Creative Commons Attribution License (CC-BY).

\section{Reviewed by:}

Rex L. Cannon, PhD, Knoxville Neurofeedback Group, Knoxville, Tennessee, USA

Randall Lyle, PhD, Mount Mercy University, Cedar Rapids, lowa, USA

\section{Introduction}

Virtual reality (VR) is a computer-generated environment that simulates a realistic experience in three-dimensional space. Typically, this illusion is accomplished by having the user wear headgear, which covers the eyes and utilizes a stereoscopic display, displaying two slightly different angles of the scene to each eye, providing the experience of depth. This, in combination with other strategies, such as parallax and shading, creates a very realistic experience. While this technology has been primarily associated with the video game industry, it is increasingly being utilized as a clinical intervention for a variety of medical and mental health concerns (Rizzo \& Koenig, 2017).

As a mental health intervention, VR has primarily been used as a sophisticated addition to exposure therapy in the treatment of phobias (e.g., fear of heights, flying, etc.; Lamson, 1994; Rothbaum et al., 1995). Because specific environments can be created in a multidimensional, controlled platform, it provides a unique opportunity to address these fearbased concerns beyond imagining a scenario or attempting to create a live experience.

In addition to specific phobias, a recent review of VR research shows that this modality is a promising intervention used in the treatment of a variety of mental health concerns, including social anxiety, posttraumatic stress disorder (PTSD), panic disorder, generalized anxiety disorder, obsessivecompulsive disorder, schizophrenia, acute and chronic pain, addictions, eating pathology, and autism (Maples-Keller, Bunnell, Kim, \& Rothbaum, 2017). As these applications gain traction, an increasing number of companies are beginning to create VR systems that interface with various bioand neuromodulation techniques.

To date, the only published study combining VR with neurofeedback utilized a single channel $(\mathrm{Cz})$ beta reward protocol in combination with VR environments as an intervention for inattention and impulsivity. Cho et al. (2004) compared the impact 
of VR with neurofeedback, VR without neurofeedback, and a control group as an intervention for 14- to 18-year-old boys with "social problems." After eight sessions, both the VR and the non-VR groups showed improvements on a continuous performance test, while the control group did not. In addition, it was noted that the VR group showed nonsignificant trends toward more improvement than the non-VR group. This study provides initial evidence that neurofeedback combined with a therapeutic VR environment can be used successfully in the treatment of mental health concerns.

The current proof-of-concept case study was designed to determine if a consumer-grade neurofeedback system (Muse) could be used in conjunction with specially designed VR environments (Positivity by Healium) to impact gamma frontal asymmetry as well as create positive changes in mood states. If successful, such an intervention could be combined with other therapeutic modalities for the treatment of depression. The intervention in this case study has two active components: gamma frontal asymmetry neurofeedback and an Open Heart VR meditation experience.

\section{Frontal Asymmetry Neurofeed back}

The frontal asymmetry model of arousal suggests that hyperactivation in left frontal areas is associated with approach behaviors and positive mood, while hyperactivation in right frontal areas is associated with withdrawal behaviors and negative mood (Sutton \& Davidson, 1997). Not surprisingly, these patterns have also demonstrated a relationship to mental health. For example, greater left-sided activation has been associated with emotional flexibility (Papousek, Reiser, Weber, Freudenthaler, \& Schulter, 2012), emotion regulation (Jackson et al., 2003), and reductions in negative affect (Tomarken, Davidson, Wheeler, \& Doss, 1992). Extreme right frontal asymmetries have been linked to depression (Thibodeau, Jorgensen, \& Kim, 2006) and social anxiety (Moscovitch et al., 2011).

Neurofeedback interventions that shift this asymmetry toward left activation (or right deactivation) have been shown to lead to improvements in mood and an alleviation of depression symptoms. For example, Baehr and colleagues used an alpha asymmetry protocol in two different sets of case studies to successfully treat depression in patients also being treated with psychotherapy (Baehr \& Baehr, 1997; Baehr, Rosenfeld, Baehr, \& Earnest, 1999). Quaedflieg et al. (2016) showed that frontal alpha asymmetry neurofeedback can result in changes in relative frontal asymmetry at rest, suggesting it is potentially a powerful tool for use in the treatment of depression. Mennella, Patron, and Palomba (2017) extended these findings by showing that frontal alpha asymmetry neurofeedback led to significant changes in resting asymmetry as well as changes in negative mood and anxiety. Other researchers have taken the same concept but applied it by rewarding left frontal beta activity while inhibiting alpha and theta activity (Hammond, 2000, 2005a, 2005b).

While much of the historical research in this area has focused on alpha asymmetries, other research has indicated that the gamma frequency band shows the strongest and most consistent relationship to frontal lobe glucose metabolism (Oakes et al., 2004) and may be an important measure of frontal activation patterns (Davidson, 2004). For example, Ramsøy, Skov, Christensen, and Stahlhut (2018) recently found that frontal gamma asymmetry was a better predictor of motivation than alpha asymmetry. Another recent study demonstrated that gamma frontal asymmetry is a reliable metric for assessing shifts in emotional states (Bonnstetter, Hebets, \& Wigton, 2015).

Utilizing the two frontal sensors on the Muse headset (AF7, AF8), StoryUp XR (Columbia, MO) in coordination with the NeuroMeditation Institute designed a gamma (30-44 Hz) frontal asymmetry protocol. The protocol provided a reward when the ratio of left frontal gamma was greater than right frontal gamma. Positive feedback was provided by the VR meditation scene advancing when criterion was met.

\section{Open Heart VR Meditation}

In addition to using frontal gamma asymmetry neurofeedback, the experience in this study incorporated a guided meditation to facilitate a positive mood state. Researchers have successfully categorized meditation styles based on how attention is directed, the intention of the meditator, and which brain waves are activated and in which brain regions (Tarrant, 2017). Based on this understanding, meditation styles that emphasize activation of a positive emotional state consistently result in increased left frontal activation and frequently in the gamma EEG band. For example, Engström presented data on a Tibetan Buddhist with many years of compassion meditation practice (Engström \& Söderfeldt, 2010). During meditation, fMRI imaging revealed activation of several brain regions involved in sustained attention and empathy. 
However, the strongest findings were related to activation of the left prefrontal cortex.

In a study comparing eight long-term meditators with eight novice meditators, there were common activation patterns found in the adepts when engaged in a compassion form of meditation. Among the findings, expert meditators showed large increases in gamma brainwave activity during the meditation in several areas of the brain including the left prefrontal cortex (Lutz, Greischar, Rawlings, Ricard, \& Davidson, 2004).

The current report examines the impact of a brief Open Heart VR experience combined with a gamma asymmetry neurofeedback protocol. Specifically, this proof-of-concept case study is designed to determine the feasibility of combining VR and brain computer interface $(\mathrm{BCl})$ to shift frontal gamma activity and positively influence mood states.
Changes in mood were assessed through self-report questionnaires (PANAS and STCl-S $<30>$ ) as well as gamma asymmetry patterns measured in the frontal lobes with SLORETA analyses.

\section{Material and Methods}

\section{Participants}

Participants were four male, Caucasian firefighters with a mean age of $39.5(S D=5.6)$. Participants self-reported their experience with meditative practices (i.e., seated meditation, yoga, qigong, chanting, prayer, or other practice) over the last 6 months and amount of time engaged in these practices each week. This information along with education level in listed in Table 1. The study was performed in a private office of a firefighter union hall. It was approved by the Quorum Institutional Review Board, Seattle, WA.

\section{Table 1}

Demographic Data

\begin{tabular}{cccccccc}
\hline $\begin{array}{c}\text { Client } \\
\#\end{array}$ & Age & Gender & Ethnicity & Education & $\begin{array}{c}\text { Meditative } \\
\text { Practice Over the } \\
\text { Last 6 Months }\end{array}$ & $\begin{array}{c}\text { Time in } \\
\text { Meditative } \\
\text { Practice } \\
\text { Weekly }\end{array}$ & $\begin{array}{c}\text { Mental Health } \\
\text { Diagnoses Within } \\
\text { Last 12 Months }\end{array}$ \\
\hline 1 & 44 & Male & White & Bachelor's & None & 0 & PTSD \\
2 & 43 & Male & White & Some College & None & 0 & Depression \\
3 & 30 & Male & White & Associate & None & 0 & None \\
4 & 41 & Male & White & High School & 1-2x/week & $1-60$ min & $\begin{array}{c}\text { Anxiety, } \\
\text { Depression, PTSD }\end{array}$ \\
\hline
\end{tabular}

a Time participant engaged in meditative practice on a weekly basis, in the last 6 months. Example of meditative practice examples in questionnaire include: seated meditation, yoga, qigong, chanting, prayer, or other practices.

${ }^{b}$ Amount of minutes spent in meditative practice each week.

\section{Intervention}

Brain Computer Interface. In an effort to make this intervention accessible, the authors sought to utilize a wireless $\mathrm{BCl}$ in combination with a wireless $\mathrm{VR}$ headset (Samsung Gear VR powered by an Android s7 phone; see Figure 1). We elected to use the Muse EEG headband as it was found to have better usability than the primary competitor in this market (Peining, Tan, \& Phyo Wai, 2017). In addition, the Muse headband has demonstrated its usefulness in mindfulness training (Bhayee et al., 2016), the ability to distinguish "pain" versus "no pain" brain states (Karydis, Foster, \& Mershin, 2016), and as a neurofeedback device to teach mental states of concentration and relaxation (Kovacevic, Ritter, Tays, Moreno, \& McIntosh, 2015).

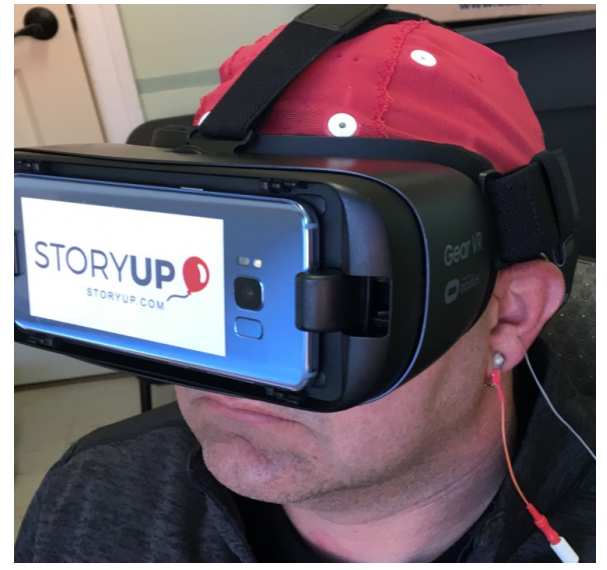

Figure 1. Participant with wireless VR headset. 
The Muse headband consists of four active electrodes at sites AF7, AF8, TP9, and TP10, with the reference position at FPZ. For the current application, software was developed by StoryUp XR and the NeuroMeditation Institute which calculated the ratio of gamma activity $(30-44 \mathrm{~Hz})$ between sites AF7 and AF8. The protocol was designed such that a relative shift in gamma power to the left electrode site resulted in a designated action in the VR experience. Electrode sites TP9 and TP10 were not utilized in this protocol design.

Virtual Reality Meditation. Positivity by Healium is a commercially available VR meditation experience designed to work in conjunction with the Muse headband. The experience is produced by StoryUp XR using 360-degree video photography. The initial scene displays a blue line graph indicating gamma asymmetry, with a red line indicating the threshold level (see Figure 2).

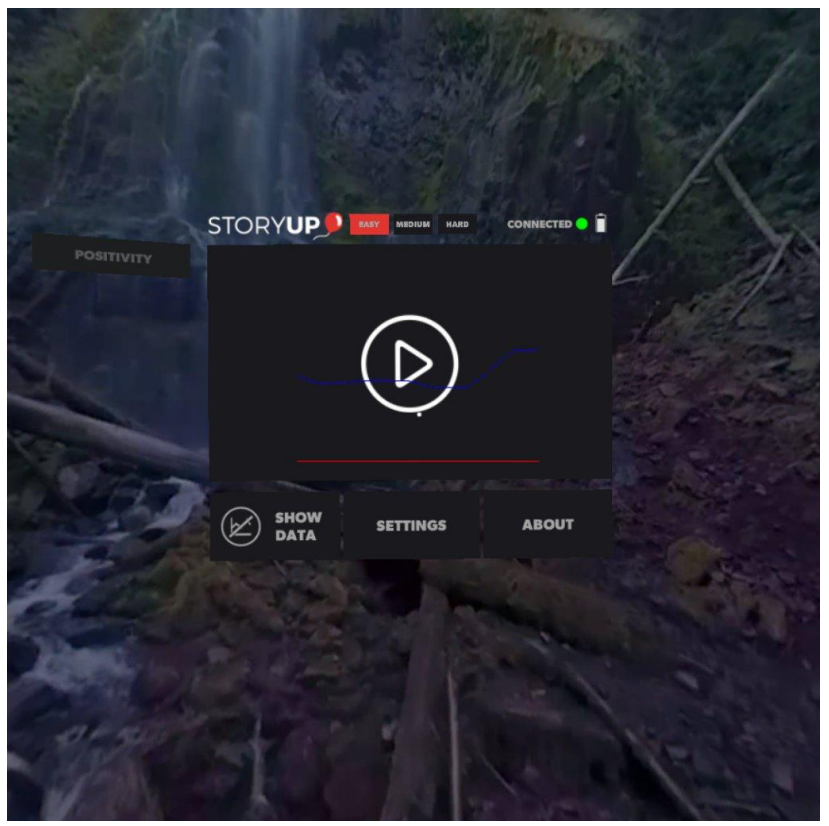

Figure 2. Initial scene in VR experience with blue line graph indicating gamma asymmetry and a red line indicating the threshold level.

Threshold can be adjusted in the VR headset, determining the degree to which left frontal gamma must be greater than right frontal gamma to meet criterion. In the current study, all subjects engaged the experience at the lowest challenge setting. Following approximately 20 seconds of calibration, the scene shifts to the base of a waterfall. There is soft piano and violin music playing in the background. Approximately 20 seconds into the experience, a woman's voice begins guiding the viewer through a positivity meditation, directing the user to recall a time in their lives when they felt grateful, appreciative, or happy. As the gamma asymmetry shifts to the left and remains above the threshold, the camera in the VR experience slowly glides up the waterfall and then back down. If at any point during the experience the gamma asymmetry indicator falls below the threshold, the scene freezes, the screen is filtered in red, and the voice returns to coach the attention back to a positive emotional state. Based on the number of stops and starts, most experiences last between four and five minutes.

\section{Measures}

Demographic Questionnaire. This questionnaire asked subjects to identify information related to their sex, age, race, education level, and history of mental illness.

Positive and Negative Affect Schedule (PANAS). This 20-item self-report scale asks subjects to identify how much they feel particular emotions in the present moment. Response options include very slightly or not at all, a little, moderately, quite a bit, or extremely, scored 1 through 5 respectively. The scale is composed of two separate mood scales, one that measures positive affect (PA) and one that measures negative affect (NA). Reliability and validity reported by Watson, Clark, and Tellegen (1988) was moderately good. For the Positive Affect Scale, the Cronbach alpha coefficient was .86 to .90; for the Negative Affect Scale, .84 to .87. Over an 8week time period, the test-retest correlations were .47-.68 for the PA and .39-.71 for the NA. The PANAS has strong reported validity with such measures as general distress and dysfunction, depression, and state anxiety.

State-Trait-Cheerfulness-Inventory $<30\rangle \quad$ (STCl$\mathbf{S}\langle 30>$ ). This 30 -item self-report scale measures three dimensions including state-cheerfulness, stateseriousness, and state-bad mood. The 4-point Likert scale of responses include strongly disagree, moderately disagree, moderately agree, and strongly agree, scored 1 through 4 respectively. The three states, or subscales, can be divided further into subclusters. State-cheerfulness clusters include cheerful and hilarity. State-seriousness includes clusters earnest, pensive, and sober. State-bad mood includes clusters sad and ill-humored. Ruch and Köhler (1999) demonstrated the scale had good internal consistency for the state-cheerfulness and state-bad mood subscales, .91 and .93, respectively. 
State-seriousness had the lowest Cronbach's $\alpha, .79$. Test-retest reliability for $\mathrm{STCl}-\mathrm{S}<30>$ (English version) is not available (Ruch \& Köhler, 1999). For the current study, only the cheerfulness and bad mood subclusters were examined.

EEG Data Collection. The EEG data in this study was sampled with 19 electrodes in the standard 1020 International placement referenced to linked ears. Data was collected for $5 \mathrm{~min}$ of baseline (eyes open), during the VR Positivity experience (4-5 $\mathrm{min})$, and 5 min postintervention (eyes open). Each raw EEG file was processed through the qEEG-Pro (qEEG Professionals, The Netherlands) Standardized Artifact Rejection Algorithm (S.A.R.A). This process removes segments from an EEG recording that are likely due to other sources, such as eye blinks, muscle tension, etc. Using an automated process such as this ensures that each file is handled in the same manner and reduces the possibility of bias in the artifact removal process. Raw files were then manually inspected. Of the 12 EEG recordings, all of the EEG samples recorded during the VR experience were eliminated due to excessive artifact (primarily eye movements). All pre-post EEG data samples were acceptable.

\section{Procedures}

The four subjects in this case study were firefighters who volunteered to test the intervention as a potential tool to assist with occupational burnout. The firefighters met at the firefighter union hall and took turns participating in the intervention in a private office. After a verbal description of the study process and completing consent forms, subjects completed a demographic form, PANAS, and STCI$S<30>$ questionnaires. Subjects were fitted with a 19-channel EEG electrocap (Electro-Cap International, Inc., Eaton, OH). Each electrode was prepared using electrogel conductance paste (Electro-Cap International, Inc., Eaton, $\mathrm{OH}$ ). Impedences for all sites were assessed and kept below $10 \mathrm{k} \Omega$. Subjects completed a 5 -min, eyesopen EEG baseline, recorded using a BrainMaster Discovery amplifier (BrainMaster Technologies, Inc., Bedford, $\mathrm{OH}$ ). Following the initial baseline recording, subjects were assisted in putting on the Muse headband and VR headgear. The subject was then instructed to simply enjoy the VR experience, follow along with the guided meditation, and attempt to keep the blue line above the red line. At the conclusion of the Positivity VR experience, the headgear was removed and a post-VR, 5-min EEG was recorded using the same instructions as the previous recordings. Following the final EEG recording, the subject completed a post-VR PANAS and STCl-S $<30>$ questionnaire.

\section{Data Analysis}

Artifact-free files were processed through BrainAvatar sLORETA analysis software (BrainMaster Technologies, Inc.) to obtain current source density (CSD) estimates for the Gamma band $(30-44 \mathrm{~Hz})$. These values were obtained for the left and right "frontal lobe" regions of interest (ROIs) as defined in the BrainAvatar Imaging software (BrainMaster Technologies, Inc., Bedford, $\mathrm{OH}$ ). Consistent with previous research utilizing these values as a measure of frontal asymmetry (see Bonnstetter et al., 2015), a left versus right frontal ROI ratio score was calculated before and after the VR intervention. This value was comprised of the average of the 1,088 left hemisphere voxels divided by the average of the 1,088 right hemisphere voxels. Values greater than 1.0 indicate greater gamma activation in the left hemisphere, while values less than 1.0 indicate greater activation in the right hemisphere. The pre-post asymmetry ratios were then compared to produce a percent of change for each subject using the following equation: (time 2 ratio - time 1 ratio) / time 2 ratio.

Self-report scale scores for the PANAS and STCI$S<30>$ were examined by comparing pre and post scores for each subject and calculating a percent of change. Percent change values were calculated by dividing the difference of specific pre and post subscales by the maximum possible score for the given subscale.

\section{Results}

\section{Subject 1}

Subject 1 began with a symmetrical gamma pattern (1.01), which shifted slightly to the left following the intervention (1.08). Similarly, most of the self-report scales demonstrated only minor shifts (see Table 2).

\section{Subject 2}

This subject began with a slight gamma asymmetry to the left, which became more prominent after the intervention, shifting from 1.10 to 1.20 , an increase of nearly $9 \%$. On the PANAS, this subject showed a $10 \%$ increase in his positive affect score as well as a $10 \%$ decrease in negative affect score. On the STCI scale, this subject demonstrated a $15 \%$ increase in state-cheerfulness and a $15 \%$ decrease on the state-bad mood scale a well as on both of the statebad mood sub clusters (i.e., sad, ill-humored). 


\section{Subject 3}

Subject 3 began with an asymmetry to the right (0.84), which moved further in this direction following the intervention (0.72). While the PANAS ratings showed a significant increase in positive affect $(10 \%)$, there was very little change in negative affect $(-4.0 \%)$. On the STCl, cheerfulness decreased by $20 \%$ while bad mood ratings stayed the same.

\section{Subject 4}

This subject began with an asymmetry to the right (0.65) but ended with a strong asymmetry to the left (1.52), a change of $133 \%$. There were only minor changes in the self-report scores on the PANAS, positive affect and negative affect decreasing by $6 \%$ and $4 \%$, respectively. On the STCl, cheerfulness increased by $15 \%$ and the ill-humored sub cluster of the state-bad mood scale decreased by $20 \%$. A visual representation of positive and negative mood in relation to gamma asymmetry is shown in Figures 3 and 4 , respectively.

\section{Table 2}

Percent Change

\begin{tabular}{|c|c|c|c|c|c|c|c|c|c|c|c|c|}
\hline & \multicolumn{3}{|c|}{ Subject 1} & \multicolumn{3}{|c|}{ Subject 2} & \multicolumn{3}{|c|}{ Subject 3} & \multicolumn{3}{|c|}{ Subject 4} \\
\hline $\begin{array}{l}\text { Gamma Ratio in } \\
\text { Frontal Lobe }^{a}\end{array}$ & 1.01 & 1.08 & $6.29 \%$ & 1.10 & 1.20 & $8.94 \%$ & 0.84 & 0.72 & $-14.70 \%$ & 0.65 & 1.52 & $133.14 \%$ \\
\hline \multicolumn{13}{|l|}{ PANAS ${ }^{b}$} \\
\hline Positive Affect & 29 & 27 & $-4.00 \%$ & 35 & 40 & $10.00 \%$ & 37 & 42 & $10.00 \%$ & 45 & 42 & $-6.00 \%$ \\
\hline \multicolumn{13}{|l|}{ STCl ${ }^{c}$} \\
\hline $\begin{array}{l}\text { State- } \\
\text { Cheerfulness }\end{array}$ & 23 & 22 & $-2.50 \%$ & 28 & 30 & $5.00 \%$ & 37 & 32 & $-12.50 \%$ & 30 & 34 & $10.00 \%$ \\
\hline Cheerful & 10 & 11 & $5.00 \%$ & 13 & 16 & $15.00 \%$ & 18 & 14 & $-20.00 \%$ & 14 & 17 & $15.00 \%$ \\
\hline Hilarity & 13 & 11 & $-10.00 \%$ & 15 & 14 & $-5.00 \%$ & 19 & 18 & $-5.00 \%$ & 16 & 17 & $5.00 \%$ \\
\hline
\end{tabular}

a Values $>1.0$ indicate greater gamma activation in the left frontal ROI. Values $<1.0$ indicate greater activation in the right frontal ROI. Pre and post values are the left to right gamma ratio in the frontal lobe.

${ }^{b}$ Maximum possible score for each PANAS subscale is positive and negative affect is 50 .

${ }^{c}$ Maximum score for STCI State-Cheerfulness is 40 with divided sub-clusters, cheerful and hilarity, equaling 20 each.

Maximum score for State-Bad Mood is 40 with divided subclusters, sad and ill-humored, equaling 20 each. 


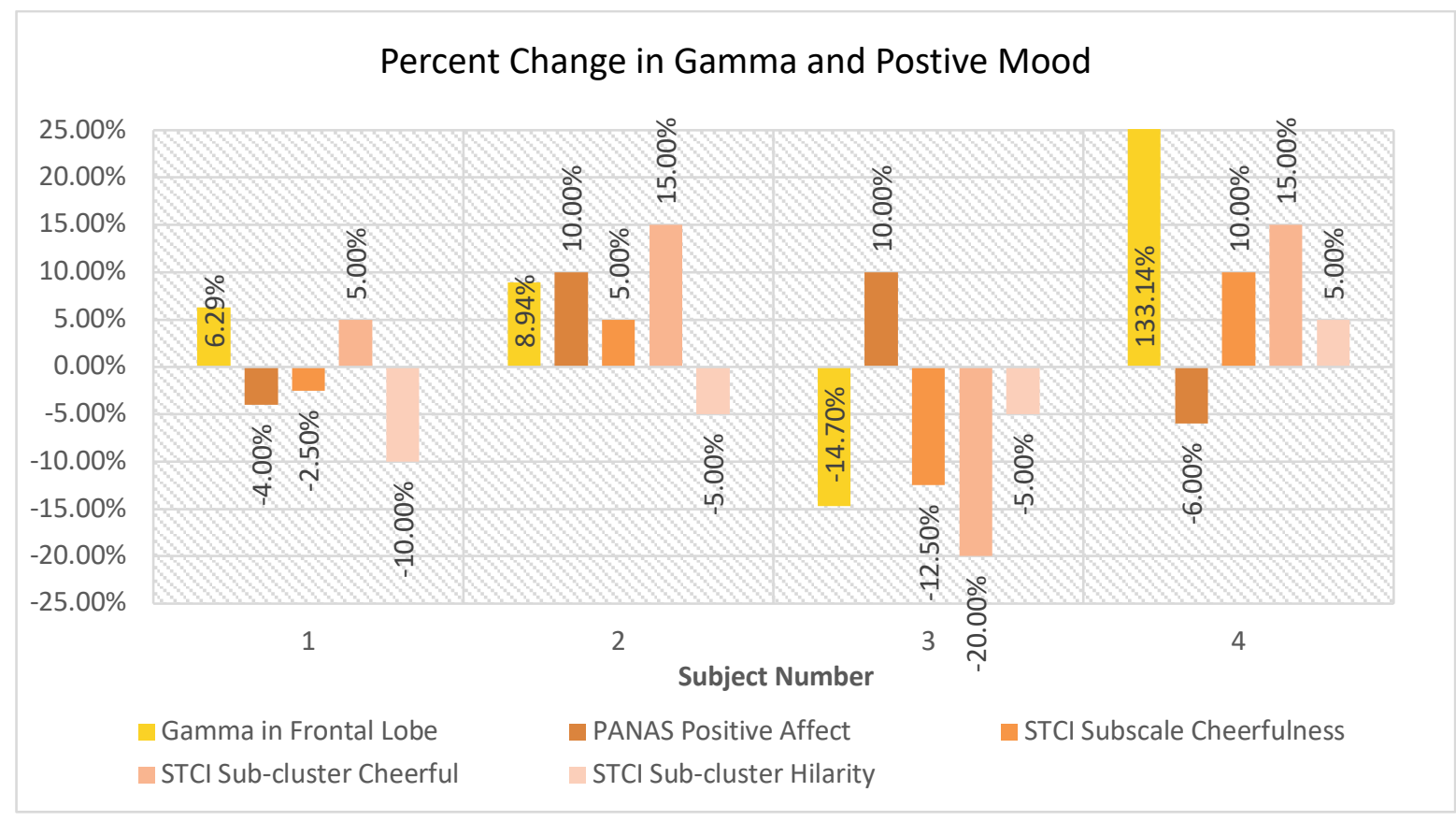

Figure 3. Percent changes of left-right gamma wave ratio and positive mood subscales of PANAS and STCI-S $<30>$ before and after VR experience.

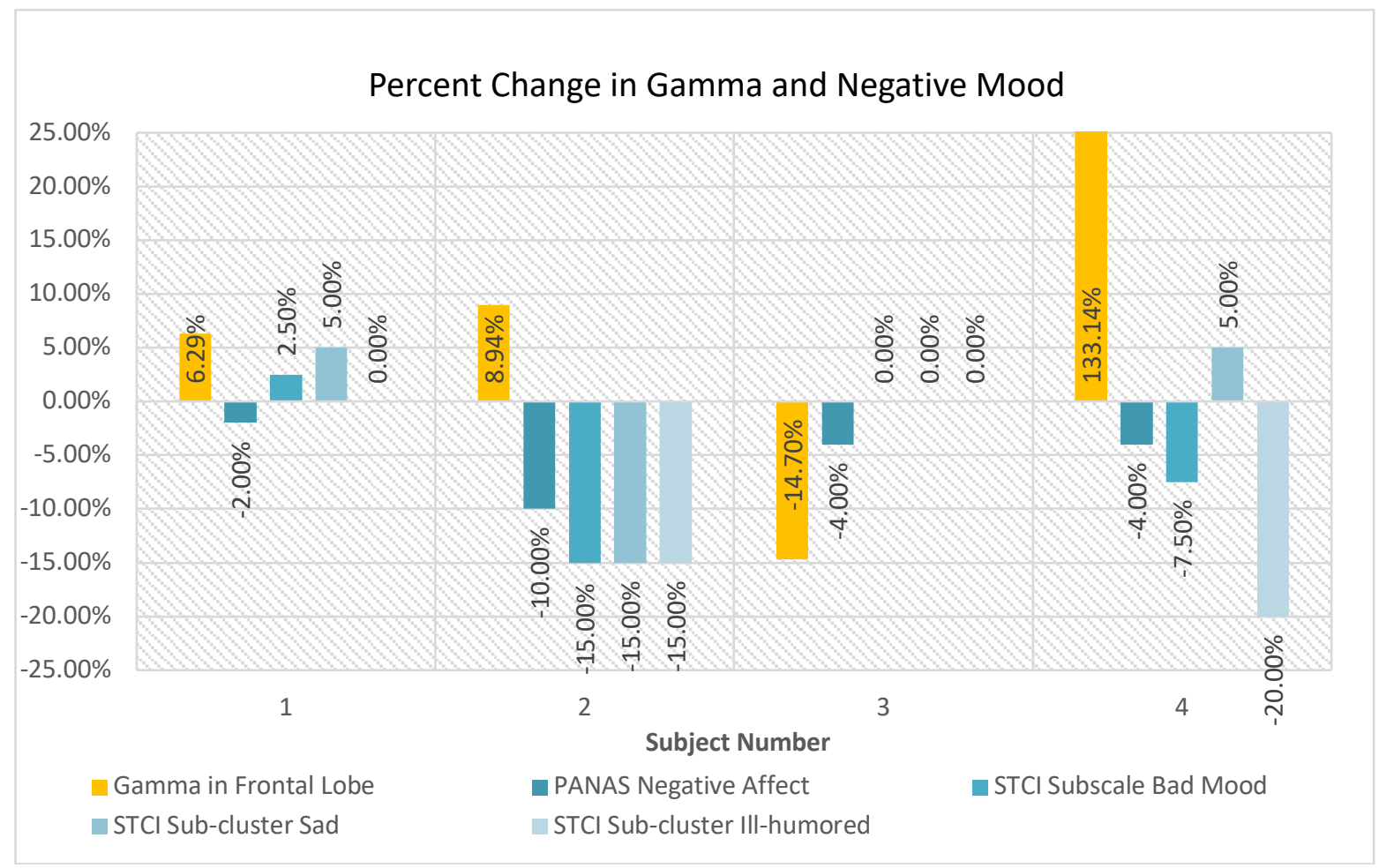

Figure 4. Percent changes of left-right gamma wave ratio and negative mood subscales of PANAS and $\mathrm{STCl}-\mathrm{S}<30>$ before and after VR experience. 


\section{Discussion}

To date, this is only the second study to examine the combination of neurofeedback with virtual reality. It was designed as a proof of concept to determine a) if such an intervention could be successfully used to administer a gamma asymmetry protocol and b) to determine if such an intervention could be linked to changes in mood.

To address the first of these considerations, it was noted that three of the four subjects showed changes in the expected direction (increased left gamma asymmetry). It is interesting that the only subject to show changes in the opposite direction (Subject 3) started with an asymmetry toward the right. While Subject 4 demonstrated that you could begin this intervention with an asymmetry on the right and end on the left, it is also possible that the intervention was not enough to shift the asymmetry for Subject 3 or it was not enough to shift the asymmetry beyond the duration of the intervention. We know that frontal asymmetry is a moderately stable condition in adults (Deldin \& Chiu, 2005), and it is possible that this subject showed a shift in asymmetry toward the left during the intervention but then returned to his baseline asymmetry as soon as the intervention ended. It is also possible that Subject 3 is experiencing some level of rumination or brooding that is interfering with his ability to fully engage with the process. A study by Barnhofer and Chittka demonstrated that subjects scoring high in brooding were unable to shift frontal asymmetry toward left-sided activation in response to a lovingkindness meditation (Barnhofer, Chittka, Nightingale, Visser, \& Crane, 2010). They speculated that the task of engaging positive emotional states was too far removed from their current state. These subjects were only successful with shifting frontal asymmetry when utilizing a focus meditation which drew their attention away from their negative thinking patterns toward a neutral stimulus (e.g., the breath). To extrapolate from these findings, it is possible that Subject 3 was unable to connect with the positivity meditation provided and may have been better served by a focus-style meditation (Tarrant, 2017). It is also possible that Subject 3's shift toward the right was influenced by other internal states or personal stressors that were not examined in this study.

The second objective of this study related to the notion that the intervention could have a positive impact on mood. A visual representation of positive and negative mood in relation to gamma asymmetry can be seen in Figures 3 and 4, respectively. While there were some small shifts in Positive and Negative Affect as measured by the PANAS, there were much greater shifts in State-Cheerfulness and State-Bad Mood as measured by the STCl-S $<30\rangle$. Three of the four subjects showed increases in Cheerfulness, while one subject (Subject 3) showed decreases in this measure. Two of the subjects demonstrated large decreases of the subcluster "IIIhumored" (Subjects 2 and 4), while no subjects demonstrated an increase. Overall, these were positive findings and provide preliminary evidence that subjects can experience an increase of positive affect and/or a decrease of negative affect following a relatively brief neurofeedback/VR experience.

While there were not enough subjects in this study to conduct statistical analyses, it seems important to note that the two subjects reporting the largest positive changes in mood states were the two that showed the largest shifts in gamma asymmetry to the left. The only subject to report decreased cheerfulness was the same subject that showed increasing right asymmetry. The subject demonstrating only minor shifts in gamma also reported only minor changes in mood. These findings suggest that the asymmetry as measured in this study is, in fact, related to changes in emotional states.

An examination of subject demographics in relation to intervention response is also of interest. Subject 4 began with a strong right asymmetry and finished with a strong left asymmetry. This is also the only subject with any regular meditation practice. This subject also acknowledged three different mental health diagnoses. It is possible that this subject has engaged in previous psychological/emotional work which has enabled him to better utilize the intervention in this study. Interestingly, the only other subject to begin with a right asymmetry, ended with a stronger right asymmetry. This subject (Subject 3), denied any mental health concerns and has no history of meditative practice. As previous research has identified that a right asymmetry is frequently associated with depressed mood and/or anxiety (Moscovitch et al., 2011; Thibodeau et al., 2006), it is possible that this subject has less awareness of their psychological/emotional state and consequently was less able to effectively engage the intervention. These observations and speculations should be investigated further in a follow-up study which includes a more detailed examination of demographics and response to the intervention. 
Overall, these results are promising as the intervention in question was only four to five minutes in length and the subjects were given very little context and minimal instruction. These results provide support for the idea that a consumer-grade $\mathrm{BCI} / \mathrm{VR}$ intervention can potentially have therapeutic utility and deserves further study. Because the technology is relatively easy to use and inexpensive, it may serve as a wellness tool in work and school environments, as a calming technique for persons receiving medical/dental procedures, or as an adjunct to traditional therapeutic interventions for anxiety or depression. In fact, such an intervention may be appropriate as "homework" for clients enrolled in traditional neurofeedback training.

In the future, it would be helpful to explore similar interventions with larger sample sizes, clearly defined subject pools (e.g., depressed, anxious), and control conditions. In addition, it would be helpful to isolate the elements of the $\mathrm{BCI} / \mathrm{VR}$ experience to more clearly define the relative contribution of each element to the overall impact. For example, viewing nature, engaging in an Open Heart meditation, and gamma asymmetry neurofeedback may each provide a unique impact on positive and negative emotional states. Because this is a relatively new and novel approach to neurofeedback, future studies with this type of experience should also include follow up questionnaires and/or participant interviews to better ascertain the internal state and reaction to elements of the VR experience. Beyond single experience research, it will also be important to examine the potential of such an intervention incorporated into a larger intervention. For example, could such an intervention be utilized as an adjunctive technique for someone enrolled in a traditional course of neuro-or psychotherapy? Could such an intervention be successfully included in a structured group experience for persons learning skills to manage anxiety or depressive symptoms?

\section{Declaration of Interests}

Jeff Tarrant, PhD, is contracted by StoryUp VR to assist in product development and assessment.

\section{References}

Baehr, E., \& Baehr, R. (1997). The use of brainwave biofeedback as an adjunctive therapeutic treatment for depression: Three case studies. Biofeedback, 25(1), 10-11.

Baehr, E., Rosenfeld, J. P., Baehr, R., \& Earnest, C. (1999). Clinical use of an alpha asymmetry protocol in treatment of mood disorders. In J. R. Evans \& A. Abarbanel (Eds.), Introduction to quantitative EEG and neurofeedback (pp. 181-201). New York: Academic Press. http://dx.doi.org /10.1016/B978-012243790-8/50009-2
Barnhofer, T., Chittka, T., Nightingale, H., Visser, C., \& Crane, C (2010). State effects of two forms of meditation on prefrontal EEG asymmetry in previously depressed individuals. Mindfulness, 1(1), 21-27. http://dx.doi.org /10.1007/s12671010-0004-7 x

Bhayee, S., Tomaszewski, P., Lee, D. H., Moffat, G., Pino, L., Moreno, S., \& Farb, N. A. S. (2016). Attentional and affective consequences of technology supported mindfulness training: A randomized, active control, efficacy trial. BMC Psychology, 4, 60. http://dx.doi.org/10.1186/s40359-016-0168-6

Bonnstetter, R. J., Hebets, D., \& Wigton, N. L. (2015). Frontal gamma asymmetry in response to soft skills stimuli: A pilot study. NeuroRegulation, 2(2), 70-85. http://dx.doi.org /10.15540/nr.2.2.70

Cho, B.-H., Kim, S., Shin, D. I., Lee, J. H., Lee, S. M., Kim, I. Y., \& Kim, S. I. (2004). Neurofeedback training with virtual reality for inattention and impulsivity. CyberPsychology \& Behavior, 7(5), 519-526. http://dx.doi.org/10.1089/cpb.2004.7.519

Davidson, R. J. (2004). What does the prefrontal cortex "do" in affect: Perspectives on frontal EEG asymmetry research. Biological Psychology, 67(1-2), 219-234. http://dx.doi.org /10.1016/j.biopsycho.2004.03.008

Deldin, P. J., \& Chiu, P. (2005). Cognitive restructuring and EEG in major depression. Biological Psychology, 70(3), 141-151. http://dx.doi.org/10.1016/j.biopsycho.2005.01.003

Engström, M., \& Söderfeldt, B. (2010). Brain activation compassion meditation: A case study. Journal of Alternative and Complementary Medicine, 16(5), 597-599. http://dx.doi.org/10.1089/acm.2009.0309

Hammond, D. C. (2000). Neurofeedback treatment of depression with the Roshi. Journal of Neurotherapy, 4(2), 45-56. http://dx.doi.org/10.1300/J184v04n02_06

Hammond, D. C. (2005a). Neurofeedback treatment of depression and anxiety. Journal of Adult Development, 12(23), 131-137. http://dx.doi.org/10.1007/s10804-005-7029-5

Hammond, D. C. (2005b). Neurofeedback with anxiety and affective disorders. Child and Adolescent Psychiatric Clinics of North America, 14(1), 105-123. http://dx.doi.org/10.1016 /j.chc. 2004.07.008

Jackson, D. C., Mueller, C. J., Dolski, I., Dalton, K. M., Nitschke, J. B., Urry, H. L., ... Davidson, R. J. (2003). Now you feel it, now you don't: Frontal brain electrical asymmetry and individual differences in emotion regulation. Psychological Science, 14(6), 612-617. http://dx.doi.org/10.1046/j.09567976.2003.psci_1473.x

Karydis, T., Foster, S. L., \& Mershin, A. (2016, June). Selfcalibrating protocols as diagnostic aids for personal medicine, neurological conditions and pain assessment. Proceedings of the 9th ACM International Conference of Pervasive Technologies Related to Assistive Environments, No. 61. http://dx.doi.org/10.1145/2910674.2935852

Kovacevic, N., Ritter, P., Tays, W., Moreno, S., \& McIntosh, A. R. (2015). 'My virtual dream': Collective neurofeedback in an immersive art environment. PLOS One, 10(7), e0130129. http://dx.doi.org/10.1371/journal.pone.0130129

Lamson, R. J. (1994). Virtual therapy of anxiety disorders. CyberEdge Journal, 4(2), 1, 6-8.

Lutz, A., Greischar, L. L., Rawlings, N. B., Ricard, M., \& Davidson, R. J. (2004). Long-term meditators self-induce high-amplitude gamma synchrony during mental practice. Proceedings of the National Academy of Sciences of the United States of America, 101(46), 16369-16373. http://dx.doi.org/10.1073 /pnas.0407401101

Maples-Keller, J. L., Bunnell, B. E., Kim, S.-J., \& Rothbaum, B. O. (2017). The use of virtual reality technology in the treatment of anxiety and other psychiatric disorders. Harvard Review of Psychiatry, 25(3), 103-113. http://dx.doi.org/10.1097 /HRP.0000000000000138

Mennella, R., Patron, E., \& Palomba, D. (2017) Frontal alpha asymmetry neurofeedback for the reduction of negative affect 
and anxiety. Behaviour Research and Therapy, 92(5), 32-40. http://dx.doi.org/10.1016/j.brat.2017.02.002

Moscovitch, D. A., Santesso, D. L., Miskovic, V., McCabe, R. E., Antony, M. M., \& Schmidt, L. A. (2011). Frontal EEG asymmetry and symptom response to cognitive behavioral therapy in patients with social anxiety disorder. Biological Psychology, $\quad 87(3), \quad 379-385 . \quad$ http://dx.doi.org/10.1016 /j.biopsycho.2011.04.009

Oakes, T. R., Pizzagalli, D. A., Hendrick, A. M., Horras, K. A., Larson, C. L., \& Abercrombie, H. C., ... Davidson, R. J. (2004). Functional coupling of simultaneous electrical and metabolic activity in the human brain. Human Brain Mapping, 21(4), 257-270. http://dx.doi.org/10.1002/hbm.20004.

Papousek, I., Reiser, E. M., Weber, B., Freudenthaler, H. H., \& Schulter, G. (2012). Frontal brain asymmetry and affective flexibility in an emotional contagion paradigm. Psychophysiology, 49(4), 489-498. http://dx.doi.org/10.1111 j.1469-8986.2011.01324.x

Peining, P., Tan, G., \& Phyo Wai, A. A. (2017, August). Evaluation of consumer-grade EEG headsets for $\mathrm{BCl}$ drone control. Presented at IRC Conference on Science, Engineering, and Technology. Retrieved from http://oar.astar.edu.sg:80/jspui/handle/123456789/2149

Quaedflieg, C. W. E. M., Smulders, F. T. Y, Meyer, T., Peeters, F., Merckelbach, H., \& Smeets, T. (2016). The validity of individual frontal alpha asymmetry EEG neurofeedback. Social Cognitive and Affective Neuroscience, 11(11), 33-43. http://dx.doi.org/10.1093/scan/nsv090

Ramsøy, T. Z., Skov, M., Christensen, M. K., \& Stahlhut, C. (2018). Frontal brain asymmetry and willingness to pPay. Frontiers in Neuroscience, 12, 138. http://dx.doi.org/10.3389 /fnins.2018.00138

Rizzo, A., \& Koenig, S. T. (2017). Is clinical virtual reality ready for

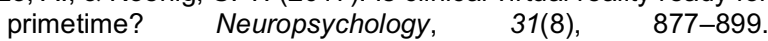
http://dx.doi.org/10.1037/neu0000405

Rothbaum, B. O., Hodges, L. F., Kooper, R., Opdyke, D., Williford, J. S., \& North, M. (1995). Effectiveness of computer- generated (virtual reality) graded exposure in the treatment of acrophobia. American Journal of Psychiatry, 152(4), 626628. http://dx.doi.org/10.1176/ajp.152.4.626

Ruch, W. \& Köhler, G. (1999). The measurement of state and trait cheerfulness. In I. Mervielde, I. Deary, F. De Fruyt, and F. Ostendorf (Eds.), Personality Psychology in Europe (Vol. 7, pp. 67-83). Tilburg, Netherlands: Tilburg University Press. https://www.uzh.ch/cmsssl/psychologie/dam/jcr:0000000038b5-2dd4-ffff-ffffe7a43322/63_m_1999_Ruch_Koehler.pdf

Sutton, S. K., \& Davidson, R. J. (1997). Prefrontal brain asymmetry: A biological substrate of the behavioral approach and inhibition systems. Psychological Science, 8(3), 204-210. http://dx.doi.org/10.1111/j.1467-9280.1997.tb00413.x

Tarrant, J. (2017). Meditation Interventions to Rewire the Brain: Integrating Neuroscience Strategies for ADHD, Anxiety, Depression, and PTSD. Eau Claire, WI: PESI Publishing \& Media.

Thibodeau, R., Jorgensen, R. S., \& Kim, S. (2006). Depression, anxiety, and resting frontal EEG asymmetry: A meta-analytic review. Journal of Abnormal Psychology, 115(4), 715-729. http://dx.doi.org/10.1037/0021-843X.115.4.715

Tomarken, A. J., Davidson, R. J., Wheeler, R. E., \& Doss, R. C. (1992). Individual differences in anterior brain asymmetry and fundamental dimensions of emotion. Journal of Personality and Social Psychology, 62(4), 676-687. http://dx.doi.org /10.1037/0022-3514.62.4.676

Watson, D., Clark, L. A., \& Tellegen, A. (1988). Development and validation of brief measures of positive and negative affect: The PANAS scales. Journal of Personality and Social Psychology, 54(6), 1063-1070. http://dx.doi.org/10.1037 /0022-3514.54.6.1063

Received: May 8, 2018

Accepted: May 15, 2018

Published: June 30, 2018 